\title{
Neumotoxicidad y hepatotoxicidad por Crotalaria pallida en el modelo de intoxicación subcrónica en ovinos
}

\author{
Pneumotoxicity and hepatotoxicity due to Crotalaria pallida in the subchronic \\ intoxication model in sheep
}

\author{
Dumar Alexander Jaramillo-Hernández ${ }^{1,5,6}$, Doris Juliette Tamayo Rojas ${ }^{2}$, Diana \\ Consuelo Cifuentes Sánchez ${ }^{3}$, Luis Carlos Veloza Angulo, ${ }^{4}$, Víctor Manuel Molina \\ Diaz $^{5}$, Luz Natalia Pedraza Castillo ${ }^{1}$, Anita Isabel Roque Rodriguez ${ }^{1}$
}

\section{Resumen}

El objetivo del estudio fue evaluar el potencial de intoxicación subcrónica de Crotalaria pallida en ovinos. Se utilizaron seis carneros, alimentados con heno y agua potable ad libitum, y $200 \mathrm{~g} / \mathrm{animal} / \mathrm{dí}$ de ración balanceada comercial. Los ovinos fueron distribuidos en un grupo control (G1) con dos animales y un grupo experimental (G2) con cuatro animales. En la dieta de G2 se incluyó $8 \mathrm{~g}$ de materia seca (MS) de C. pallida por

\footnotetext{
${ }^{1}$ Escuela de Ciencias Animales, Facultad de Ciencias Agropecuarias y Recursos Naturales, Universidad de los Llanos, Villavicencio, Meta, Colombia

${ }^{2}$ Grupo de Investigación en Farmacología Experimental y Medicina Interna, Universidad de los Llanos, Villavicencio, Meta, Colombia

${ }^{3}$ Clínica de las Intoxicaciones y Plantas Tóxicas, Universidade Estadual Paulista Júlio de Mesquita filho. São Paulo, Brasil

${ }^{4}$ Facultad de Medicina Veterinaria y Zootecnia, Laboratorio de Patología, Universidad CES, Antioquia, Colombia

${ }^{5}$ Boehringer Ingelheim, ROPU Surámerica, Technical Services. Ingelheim, Alemania

${ }^{6}$ E-mail: dumar.jaramillo@unillanos.edu.co
}

Fuente financiera: Laboratorio de Farmacología de la Escuela de Ciencias Animales y Centro Clínico Veterinario, Facultad de Ciencias Agropecuarias y Recursos Naturales, Universidad de los Llanos. Colombia

Recibido: 22 de marzo de 2021

Aceptado para publicación: 15 de octubre de 2021

Publicado: 22 de diciembre de 2021

CLos autores. Este artículo es publicado por la Rev Inv Vet Perú de la Facultad de Medicina Veterinaria, Universidad Nacional Mayor de San Marcos. Este es un artículo de acceso abierto, distribuido bajo los términos de la licencia Creative Commons Atribución 4.0 Internacional (CC BY 4.0) [https:// creativecommons.org/licenses/by/4.0/deed.es] que permite el uso, distribución y reproducción en cualquier medio, siempre que la obra original sea debidamente citada de su fuente original 
kilogramo de peso vivo durante 28 días. Todos los animales fueron evaluados clínicamente $\mathrm{c} / 24 \mathrm{~h}$. Se les pesó y se tomaron muestras de sangre para hemograma y perfil hepático y renal en los días 0,14 y 28 posexposición a $C$. pallida. Los animales fueron sacrificados al final del estudio para la toma de muestras para análisis histopatológico. No se encontraron diferencias significativas entre grupos con respecto al examen clínico, hemograma y bioquímica sanguínea; sin embargo, en el estudio histopatológico se evidenció engrosamiento moderado de los septos alveolares en pulmón, cambio vacuolar severo en hígado y proteinuria moderada en riñón en los animales expuestos a C. pallida.

Palabras clave: alcaloides pirrolizidinicos, monocrotalina, plantas tóxicas, toxicidad

\section{AbSTRACT}

The aim of this study was to evaluate the potential for subchronic intoxication of Crotalaria pallida in sheep. Six rams were used, fed with hay and drinking water ad libitum, and $200 \mathrm{~g} /$ animal/day of commercial balanced ration. The sheep were distributed into a control group (G1) with two animals and an experimental group (G2) with four animals. In the $\mathrm{G} 2$ diet, $8 \mathrm{~g}$ of dry matter (DM) of $C$. pallida per kilogram of body weight were included for 28 days. All animals were clinically evaluated every $24 \mathrm{~h}$. Animals were weighed and blood samples were taken for hemogram and liver and kidney profile on days 0,14 and 28 post-exposure to $C$. pallida. All animals were slaughtered at the end of the study and samples were collected for histopathological analysis. No significant differences were found between groups with respect to clinical examination, hemogram data and blood chemistry; however, the histopathological study showed moderate thickening of the alveolar septa in the lung, severe vacuolar change in the liver, and moderate proteinuria in the kidney in the animals exposed to C. pallida.

Key words: pyrrolizidine alkaloids, monocrotaline, toxic plants, toxicity

\section{INTRODUCCIÓN}

Las plantas de género Crotalaria pertenecen a la familia Fabaceae que comprende cerca de 600 especies, mayormente distribuidas en los trópicos y subtrópicos (Polhill, 1981; Chong et al., 2009). En Colombia se han reportado 19 especies, en su mayoría situadas en zonas cálidas y templadas por debajo de los $3000 \mathrm{msnm}$ (Bernal, 1986). Se le utiliza mayormente en la agricultura, como abono verde y como cubierta en la preparación del suelo por su alta capacidad de fijación del nitrógeno (Mosjidis y Wang 2011). La planta es consumida accidentalmente al quedar incluida en el forraje ofrecido a los animales (Souza et al., 1997).
Plantas del género Crotalaria contienen metabolitos secundarios de naturaleza nitrogenada denominados alcaloides pirrolizidinicos (AP), tanto en hojas y florescencias, como en las semillas contenidas en vainas (Bernal, 1986), siendo entre los más relevantes en $C$. pallida la monocrotalina $(\mathrm{Mn})$ y la usaramina (Um) (Copple et al., 2004; Edgar et al., 2011). Estos compuestos son metabolizados por enzimas hepáticas tipo citocromo P-450 (CP450) originando agentes alquilantes electrofílicos tipo esteres pirrólicos (EP) (Mingatto et al., 2007). A pesar de tener una vida media de escasos segundos en un medio acuoso, estos pirroles son un poderoso agente alquilante que se une al ADN, RNA y las 
proteínas (Niwa et al., 1991; Wagner et al., 1993; Yan y Huxtable, 1995), con la subsiguiente actividad citotóxica, antimitótica y megalocítica característica de sus procesos tóxicos (Hincks et al., 1991).

Las especies tóxicas de Crotalaria poseen un rendimiento de AP entre 0.2 y $3 \%$ (Peterson y Culvenor, 1983). En Colombia han identificado AP en C. pallida en $0.18 \%$ de su materia seca (MS) y dentro de estos, la Um en una concentración de $0.16 \%$ (Díaz et al., 2014). Anjos et al. (2010) demostró que las ovejas son susceptibles a la intoxicación aguda por Mn y que la intoxicación ocurre después de una sola dosis oral $(205.2 \mathrm{mg} / \mathrm{kg}$ ); sin embargo, las ovejas desarrollan una fuerte resistencia a la Mn después de la ingestión diaria de dosis no tóxicas $(\leq 136.8 \mathrm{mg} / \mathrm{kg})$.

Según Nobre et al. (2005, las ovejas son más resistentes a la intoxicación por AP que el ganado vacuno y equino. En Brasil se han descrito cuadros de intoxicación aguda de forma experimental en ovejas con dosis entre 2.5 y $40 \mathrm{~g} / \mathrm{kg} / \mathrm{d}$ de hojas verdes de $C$. pallida (Borelli et al., 2016), además de un brote de intoxicación en Rio Grande do Norte (Riet-Correa et al., 2011). No obstante, se dispone de escasos estudios toxicológicos clínicos en rumiantes sobre la oferta ambiental asociada a plantas en Colombia, toda vez que la mayoría de los estudios se centran en la determinación de grupos de metabolitos secundarios de plantas que se asocian con eventos tóxicos en animales (Díaz, 2010, 2011, 2015; Lozano et al., 2010; Lozano y Díaz, 2013). Asimismo, son importantes los estudios sobre bioacumulación de xenobióticos en tejidos animales que son fuente de alimentos a los humanos (Díaz et al., 2014; Dusemund et al., 2018). Es así, que el objetivo de esta investigación fue determinar la posibilidad de toxicidad subcrónica en ovejas expuestas al consumo diario de MS de C. pallida colectada en los Llanos Orientales colombianos.
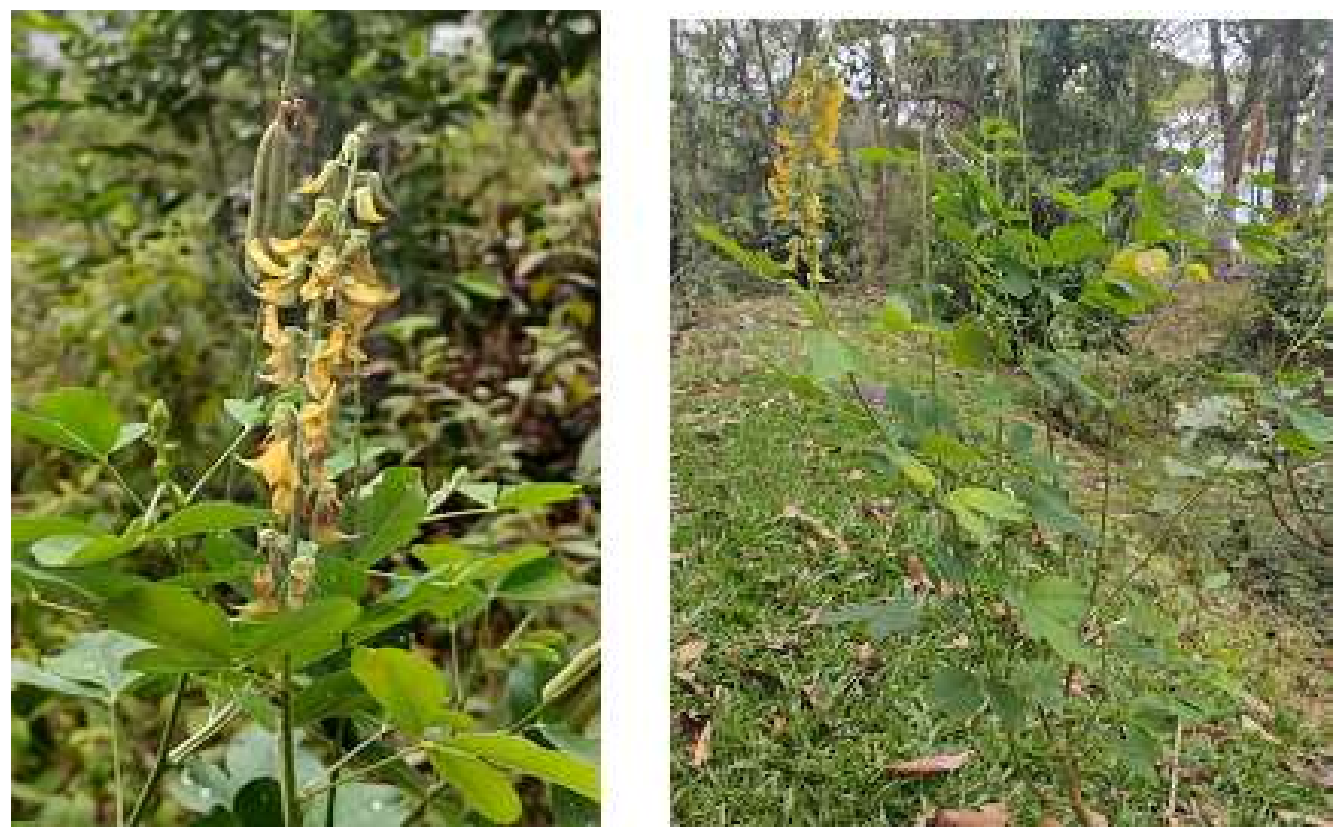

Figura 1. Crotalaria pallida 


\section{Materiales y Métodoos}

\section{Colecta y Preparado del Material Vegetal}

La planta C. pallida (Figura 1) se colectó en el departamento del Meta (Colombia), zona de piedemonte llanero del municipio de Villavicencio. La zona tiene una altitud de $465 \mathrm{msnm}$, temperatura promedio de $27^{\circ} \mathrm{C}$ y precipitación de 1900-2300 mm/año. La colecta se efectuó entre los meses de febrero a marzo, época de sequía en la zona.

Una muestra de hojas, tallo, florescencia y vainas se envió al Herbario Nacional de Colombia para la clasificación taxonómica, reportando la especie Crotalaria pallida Aiton. Las partes aéreas de la planta (hojas y pequeños tallos) fueron extraídas, limpiadas y pesadas (materia verde, MV), para luego ser sometidas en un horno de secado de aire recirculante a $60{ }^{\circ} \mathrm{C}$ durante $72 \mathrm{~h}$. El rendimiento de MS fue de $55.28 \%$. La MS fue molida hasta obtener un cernido homogéneo, guardándose a temperatura ambiente en frascos sellados.

\section{Animales y Modelo de Toxicidad Subcró- nica}

Se utilizaron seis carneros enteros, raza criollo colombianos, provenientes del sistema de producción ovino de la Granja Barcelona (Universidad de los Llanos, Colombia). Los animales tenían entre 6 y 12 meses, peso promedio $27 \mathrm{~kg}$. Se les realizó exámenes clínicos diarios durante 8 días antes del inicio de la exposición a $C$. pallida; además de pruebas paraclínicas (hemograma, coproparasitológicos y bioquímica sérica [aspartato aminotransferasa -AST-, gamma glutamil transpeptidasa -GGT-, nitrógeno ureico en sangre -BUN- y creatinina]) para confirmar su adecuado estado de salud para este estudio.

Se colocaron dos ovinos por corral dentro de la zona de experimentación del Centro Clínico Veterinario de la Universidad de los
Llanos, donde fueron alimentados con heno de Dichantium aristatum y agua potable ad libitum, y $200 \mathrm{~g} /$ animal/día de ración balanceada comercial. Luego de ocho días de adaptación, fueron divididos al azar en dos grupos: Control (G1) con dos ovinos y Grupo Experimental (G2) con cuatro ovinos. A los carneros del G2 se les incluyó en su dieta diaria $4.79 \%$ peso/peso de MV C. pallida ( 8 g MS C. pallida $/ \mathrm{kg}$ ), lo que corresponde al $1 \%$ de inclusión en la dieta (dosis baja) de acuerdo con Barbosa-Ferreira et al. (2011) durante los 28 días que duró el modelo subcrónico de intoxicación dietaria (Madingou et al., 2016). En el estudio se garantizó el bienestar de los animales experimentales cumpliendo con la normatividad nacional, Ley 84 de 1989 «Estatuto Nacional de Protección de los Animales»y y las buenas prácticas de experimentación animal asociadas a «Ethical Guidelines for the Use of Animals in Research» (NENT, 2018). Además, se contó con el aval del Centro Clínico Veterinario de la Universidad de los Llanos para el uso de los animales experimentales.

\section{Muestras y Procedimientos}

Todos los animales fueron evaluados clínicamente cada 24 h (10:00 h) desde el día de la inclusión de $C$. pallida en la dieta (día $0, \mathrm{~T} 0$ ). Se les pesó y se colectaron muestras de sangre por punción yugular, en tubo con y sin EDTA para hemoleucograma, perfil hepático (AST y GGT) y perfil renal (BUN y creatinina) los días 0 (T0), 14 (T1) y 28 (T2) pos-exposición (PE) a C. pallida. Los carneros fueron sacrificados el día 28 PE siguiendo el protocolo de Woods (2017) para proceder con la necropsia, examen anatomopatológico (Johnson y Libal, 1986). Se tomaron muestras según metodología descrita por Luna (1968) de órganos del sistema nervioso central, tracto gastrointestinal y glándulas anexas, sistema cardiovascular, sistema respiratorio y sistema reproductivo. 
Cuadro 1. Evaluación clínica de carneros sometidos a una dieta control (G1) y una dieta experimental con $1 \%$ de inclusión de Crotalaria pallida $(\mathrm{G} 2)$

\begin{tabular}{lcc}
\hline & G1 & G2 \\
\hline $\begin{array}{l}\text { Condición } \\
\text { corporal (1-5) }\end{array}$ & $3 \pm 0.2$ & $3 \pm 0.7$ \\
$\begin{array}{l}\text { Temperatura } \\
\text { rectal, }{ }^{\circ} \mathrm{C}\end{array}$ & $38.7 \pm 0.12$ & $38.5 \pm 0.23$ \\
$\begin{array}{l}\text { Frecuencia } \\
\text { cardiaca, lpm }\end{array}$ & $70 \pm 5$ & $72 \pm 5.5$ \\
$\begin{array}{l}\text { Frecuencia } \\
\text { respiratoria, rpm }\end{array}$ & $12 \pm 2$ & $11 \pm 3$ \\
\begin{tabular}{l} 
Movimientos $_{\text {ruminales }}{ }^{1}(\mathrm{n})$ \\
\hline
\end{tabular} & $2 \pm 0.4$ & $2 \pm 0.4$ \\
\hline
\end{tabular}

${ }^{1} \mathrm{En} 3 \mathrm{~min}$

No hubo diferencias estadísticas significativas entre los valores de G1 y G2 ( $p>0.05$ )

\section{Análisis Estadístico}

Se aplicó la prueba de homocedasticidad de Barttle a los datos obtenidos sobre el examen clínico y las pruebas paraclínicas. Los resultados de los grupos G1 y G2 fueron comparados mediante la prueba de Chi-cuadrado, asumiendo un nivel de significancia estadística de $\mathrm{p}<0.05$. Los hallazgos anatomopatológicos e histopatológicos se describieron según sus lesiones y análisis porcentual. Se utilizó el programa Open Stat 4® v. 9.0.

\section{Resultados}

\section{Evaluación Clínica}

En la evaluación diaria no se observaron cambios significativos ( $\mathrm{p}>0.05)$ en los signos clínicos entre los grupos G1 y G2 (Cuadro 1). Todos los animales presentaron durante los 28 días del estudio un estado mental de alerta y de docilidad, así como membra- nas mucosas de color rosa pálido. Asimismo, los movimientos ruminales variaron en frecuencia e intensidad (completos e incompletos) sin diferencias importantes entre grupos $(\mathrm{p}>0.05)$. Durante la fase experimental, los animales ganaron $35.7 \pm 3.6$ y $33.9 \pm 5.6 \mathrm{~g} / \mathrm{d}$ de peso vivo en G1 y G2, respectivamente.

Los resultados de laboratorio tuvieron una tendencia similar al examen clínico, sin diferencias significativas entre G1 y G2 ( $>0.05)$ (cuadros 2-4)

\section{Hallazgos Anatómicos e Histopatoló- gicos}

A la evaluación macroscópica de los órganos en estudio no se evidenció cambios importantes entre los animales de los grupos G1 y G2, siendo la ubicación, tamaño, peso, forma, bordes, superficie externa (lisa, brillante, trasparente), consistencia al corte y demás características de acuerdo con lo esperado para la especie.

El estudio histopatológico de los animales del grupo G1 no reportó ninguna alteración evidente en los órganos colectados, mientras que en todos los animales del grupo G2 se observaron cambios histopatológicos en hígado, pulmón y riñón. En el parénquima hepático se encontraron hepatocitos hinchados o vacuolados, cambio vacuolar severo de presentación difusa en zona media, con una leve congestión centrolobulillar, en el parénquima pulmonar engrosamiento moderado de los septos alveolares de forma generalizada (Figura 2) y proteinuria moderada en el riñón.

\section{Discusión}

Se reporta el impacto que tiene el consumo de plantas tóxicas por animales de abasto, especialmente en casos de toxicosis aguda o crónica, donde se presenta mortalidad, disminución de los parámetros productivos y gastos ocasionados por tratamientos preventi- 
Cuadro 2. Valores de la serie roja del hemograma en carneros sometidos a una dieta experimental contenido Crotalaria pallida durante 28 días

\begin{tabular}{|c|c|c|c|c|}
\hline \multirow[t]{2}{*}{ Tiempos } & \multirow[t]{2}{*}{ Parámetro } & $\begin{array}{c}\mathrm{G} 1 \\
(\mathrm{n}=2)\end{array}$ & $\begin{array}{c}\mathrm{G} 2 \\
(\mathrm{n}=4)\end{array}$ & \multirow{2}{*}{$\begin{array}{c}\text { Rango } \\
\text { fisiológico }\end{array}$} \\
\hline & & $\overline{\mathrm{x}} \quad \mathrm{DS}$ & $\overline{\mathrm{x}} \mathrm{DS}$ & \\
\hline \multirow[t]{3}{*}{ T0 } & Eritrocitos x $10^{6} / \mu 1$ & $8.9 \pm 0.12$ & $8.6 \pm 0.57$ & $9-15$ \\
\hline & Hematocrito, $\%$ & $26.75 \pm 0.7$ & $27.12 \pm 2.63$ & $27-45$ \\
\hline & Plaquetas $\times 10^{3} / \mu 1$ & $507.5 \pm 116.3$ & $572.75 \pm 122.19$ & $100-800$ \\
\hline \multirow[t]{3}{*}{$\mathrm{T} 1$} & Eritrocitos x $10^{6} / \mu 1$ & $9.8 \pm 2.0$ & $9.18 \pm 0.49$ & $9-15$ \\
\hline & Hematocrito, $\%$ & $29 \pm 2.8$ & $29.25 \pm 3.30$ & $27-45$ \\
\hline & Plaquetas $\times 10^{3} / \mu 1$ & $650 \pm 70.7$ & $539.5 \pm 109.9$ & $100-800$ \\
\hline \multirow[t]{3}{*}{$\mathrm{T} 2$} & Eritrocitos x $10^{6} / \mu 1$ & $11.7 \pm 0.4$ & $12.6 \pm 0.42$ & $9-15$ \\
\hline & Hematocrito, \% & $28.1 \pm 2.0$ & $29.75 \pm 3.52$ & $27-45$ \\
\hline & Plaquetas $\times 10^{3} / \mu 1$ & $425 \pm 106.1$ & $502.5 \pm 98.11$ & $100-800$ \\
\hline
\end{tabular}

${ }^{1}$ Radostits et al. (2000)

G1: Dieta control; G2: $1 \%$ de inclusión de Crotalaria pallida en la dieta

T0: tiempo cero (antes de administrar C. pallida al G2); T1 y T2, 14 y 28 días después de la administración diaria de C. pallida a $\mathrm{G} 2$

$\overline{\mathrm{X}}$ media estadística, DS desviación estándar

No hubo diferencias estadísticas significativas entre los valores de G1 y G2 ( $p>0.05)$

vos y/o curativos (Grosso et al., 2011). La detección de plantas tóxicas en las dietas de animales es un desafío, ya que la toxicosis no se detecta cuando es subclínica (Scasta et al., 2020).

Los animales, en muchos casos, son selectivos y evitan la ingesta de plantas tóxicas o tienen mecanismos fisiológicos que pueden contrarrestar los efectos tóxicos (Iason y Villalva, 2006): sin embargo, a pesar de ser considerado un proceso de aprendizaje denominado aversión condicionada (Provenza et al., 1992), los casos de intoxicación por plantas son frecuentes, causando pérdidas económicas a los productores (Torres, 1983; Nielsen y James, 1992; Soares et al., 2018).

La identificación de signos clínicos asociados a intoxicación por plantas puede ser una herramienta bien aplicada en el campo productivo y determinar la tolerancia de los animales permite construir planes preventivos que limiten la exposición. En este sentido, la valoración física y fisiológica de los animales de este estudio sometidos a una dieta de 8 g MS $/ \mathrm{kg}$ PV de C. pallida por 28 días no mostró alteración de parámetros clínicos ni en los parámetros paraclínicos respecto a los valores normales para la especie reportados por Radostis et al. (2000) (cuadros 1-4).

Borelli et al. (2016) describe que en la exposición a partir de 2.5 y hasta de $40 \mathrm{~g}$ $\mathrm{MV} / \mathrm{kg}$ PV de hojas de C. pallida en ovinos ocasiona la presencia de signos clínicos (diarrea, inquietud, agitación, anorexia, respiración abdominal, taquipnea y taquicardia) y muerte entre 12-21 horas. Asimismo, Laws (1968) indicó que la dosis letal para ovejas puede estar entre 11.4 y $13.6 \mathrm{~g} \mathrm{MV}$ de hojas/ $\mathrm{kg}$ PV, en tanto que Tokarnia y Döbereiner (1982) reportaron dosis tóxicas de $C$. anagyroides para rumiantes de $25 \mathrm{~g} / \mathrm{kg}$ como dosis única. 
Cuadro 3. Valores de la serie blanca del hemograma en carneros sometidos a una dieta experimental contenido Crotalaria pallida durante 28 días

\begin{tabular}{|c|c|c|c|c|c|}
\hline \multirow[t]{2}{*}{ Tiempos } & \multirow{2}{*}{$\begin{array}{l}\text { Parámetro } \\
\left(\mathrm{x} 10^{3} / \mu 1\right)\end{array}$} & $\begin{array}{c}\mathrm{G} 1 \\
(\mathrm{n}=2)\end{array}$ & \multicolumn{2}{|c|}{$\begin{array}{c}\mathrm{G} 2 \\
(\mathrm{n}=4)\end{array}$} & \multirow{2}{*}{$\begin{array}{c}\text { Rango } \\
\text { fisiológico }\end{array}$} \\
\hline & & $\overline{\mathrm{x}} \quad \mathrm{DS}$ & $\overline{\mathrm{X}}$ & DS & \\
\hline \multirow[t]{5}{*}{ T0 } & Leucocitos & $8.5 \pm 2.40$ & 9.3 & \pm 1.79 & $4-12$ \\
\hline & Neutrófilos & $2.6 \pm 0.38$ & 3.4 & \pm 1.84 & $0.7-6$ \\
\hline & Linfocitos & $4.9 \pm 1.73$ & 4.9 & \pm 0.51 & $2-9$ \\
\hline & Eosinófilos & $0.9 \pm 0.09$ & 0.76 & \pm 0.21 & $0-1$ \\
\hline & Monocitos & $0.039 \pm 0.02$ & 0.059 & \pm 0.012 & $0-0.75$ \\
\hline \multirow[t]{5}{*}{$\mathrm{T} 1$} & Leucocitos & $9.85 \pm 0.07$ & 9.6 & \pm 2.13 & $4-12$ \\
\hline & Neutrófilos & $3.6 \pm 0.11$ & 3.3 & \pm 0.9 & $0.7-6$ \\
\hline & Linfocitos & $5.6 \pm 0.91$ & 5.7 & \pm 0.6 & $2-9$ \\
\hline & Eosinófilos & $0.64 \pm 0.07$ & 0.55 & \pm 0.24 & $0-1$ \\
\hline & Monocitos & $0.049 \pm 0.06$ & 0.059 & \pm 0.02 & $0-0.75$ \\
\hline \multirow[t]{5}{*}{$\mathrm{T} 2$} & Leucocitos & $10.85 \pm 0.07$ & 9.75 & \pm 0.92 & $4-12$ \\
\hline & Neutrófilos & $4.5 \pm 0.06$ & 3.8 & \pm 0.76 & $0.7-6$ \\
\hline & Linfocitos & $5.69 \pm 0.88$ & 5.2 & \pm 0.56 & $2-9$ \\
\hline & Eosinófilos & $0.49 \pm 0.08$ & 0.39 & \pm 0.12 & $0-1$ \\
\hline & Monocitos & $0.054 \pm 0.08$ & 0.058 & \pm 0.07 & $0-0.75$ \\
\hline
\end{tabular}

${ }^{1}$ Radostits et al. (2000)

G1: Dieta control; G2: 1\% de inclusión de Crotalaria pallida en la dieta

T0: tiempo cero (antes de administrar $C$. pallida al G2); T1 y T2, 14 y 28 días después de la administración diaria de C. pallida a G2

$\bar{x}$ media estadística, DS desviación estándar

En contraste con el presente estudio, Boghossian et al. (2007) encontraron signos clínicos como pulso venoso positivo en bovinos intoxicados con C. pallida y disminución de los movimientos ruminales 59 días después de haber iniciado el experimento. Por otro lado, en estudios con otras especies de Crotalaria, por ejemplo, con C. retusa, se reportan signos neurológicos como incoordinación, depresión, anorexia y recumbencia en ovejas a las $48 \mathrm{~h}$ tras una única dosis diaria de $10 \mathrm{~g} / \mathrm{kg}$ (Nobre et al., 2005), fotosensibilización (Nobre et al., 2004a); del mismo modo, con $C$. anagyroides se reporta excitación o depresión y muerte (Tokarnia y Döbereiner, 1983).
Los resultados del hemograma y la bioquímica sanguínea del presente estudio (cuadros 2-4) estuvieron dentro de los rangos esperados para la especie (Radostits et al., 2000). Queiroz (2013), por otro lado, tampoco encontró cambios significativos en el hemograma asociados a la intoxicación con C. incana en bovinos. Asimismo, Laws (1968) tampoco reportó alteraciones en la bioquímica sanguínea asociadas a la intoxicación con C. pallida en ovejas, ni Pessoa et al. (2013) en la actividad de AST ni GGT con $C$. juncea en burros. Por el contrario, Nobre et al. (2004a) reportan elevación de GGT en bovinos intoxicados naturalmente por C. retusa. 
Cuadro 4. Química sanguínea en carneros sometidos a una dieta experimental contenido Crotalaria pallida durante 28 días

\begin{tabular}{|c|c|c|c|c|}
\hline Tiempos & Parámetro & $\begin{array}{c}\mathrm{G} 1 \\
\overline{\mathrm{x}} \pm \mathrm{DS}\end{array}$ & $\begin{array}{c}\mathrm{G} 2 \\
\overline{\mathrm{x}} \pm \mathrm{DS}\end{array}$ & $\begin{array}{l}\text { Rango } \\
\text { fisiológico }\end{array}$ \\
\hline \multirow[t]{4}{*}{ T0 } & $\begin{array}{l}\text { AST (aspartato aminotransferasa), } \\
\mathrm{U} / \mathrm{L}\end{array}$ & $85 \pm 4.24$ & $74.3 \pm 13.9$ & $60-280$ \\
\hline & $\begin{array}{l}\text { GGT (gamma glutamil } \\
\text { transpeptidasa), U/L }\end{array}$ & $40.5 \pm 1.9$ & $42.7 \pm 9.2$ & $20-52$ \\
\hline & $\begin{array}{l}\text { BUN (nitrógeno ureico en } \\
\text { sangre), } \mathrm{mg} / \mathrm{dl}\end{array}$ & $15.3 \pm 1.07$ & $12.9 \pm 4.2$ & $10-35$ \\
\hline & Creatinina, mg/dl & $1.1 \pm 0.32$ & $1.1 \pm 0.4$ & $1.2-1.9$ \\
\hline \multirow[t]{4}{*}{$\mathrm{T} 1$} & $\begin{array}{l}\text { AST (aspartato aminotransferasa), } \\
\text { U/L }\end{array}$ & $97.75 \pm 8.17$ & $105.3 \pm 18.5$ & $60-280$ \\
\hline & $\begin{array}{l}\text { GGT (gamma glutamil } \\
\text { transpeptidasa), } \mathrm{U} / \mathrm{L}\end{array}$ & $43.5 \pm 0.95$ & $43.8 \pm 2.9$ & $20-52$ \\
\hline & $\begin{array}{l}\text { BUN (nitrógeno ureico en } \\
\text { sangre), } \mathrm{mg} / \mathrm{dl}\end{array}$ & $2.65 \pm 0.64$ & $3.6 \pm 0.6$ & $10-35$ \\
\hline & Creatinina, mg/dl & $1.13 \pm 0.08$ & $1.1 \pm 0.1$ & $1.2-1.9$ \\
\hline \multirow[t]{4}{*}{$\mathrm{T} 3$} & $\begin{array}{l}\text { AST (aspartato aminotransferasa), } \\
\text { U/L }\end{array}$ & $80.9 \pm 3.27$ & $73.3 \pm 7.5$ & $60-280$ \\
\hline & $\begin{array}{l}\text { GGT (gamma glutamil } \\
\text { transpeptidasa), U/L }\end{array}$ & $40.9 \pm 3.0$ & $47.5 \pm 11.2$ & $20-52$ \\
\hline & $\begin{array}{l}\text { BUN (nitrógeno ureico en } \\
\text { sangre), } \mathrm{mg} / \mathrm{dl}\end{array}$ & $11.9 \pm 1.33$ & $12.1 \pm 1.8$ & $10-35$ \\
\hline & Creatinina, mg/dl & $1.3 \pm 0.01$ & $1.4 \pm 0.1$ & $1.2-1.9$ \\
\hline
\end{tabular}

${ }^{1}$ Radostits et al. (2000)

G1: Dieta control; G2: $1 \%$ de inclusión de Crotalaria pallida en la dieta

T0: tiempo cero (antes de administrar C. pallida al G2); T1 y T2, 14 y 28 días después de la administración diaria de $C$. pallida a $\mathrm{G} 2$

$\overline{\mathrm{x}}$ media estadística, DS desviación estándar

No hubo diferencias estadísticas significativas entre los valores de G1 y G2 ( $p>0.05)$

Los hallazgos histopatológicos reportados en este estudio coinciden con las observaciones hechas por Copple et al. (2004), quienes también encontraron vacuolización y necrosis de hepatocitos (Figura 2A) en animales expuesto a $C$. pallida. Se hipotetiza que el alcaloide $\mathrm{Mn}$ bioactivo produce alteraciones en las células endoteliales sinusoidales hepáticas que conducen a hemorragia, deposición de fibrina, lesiones hepáticas coagulativas en las células del parénquima, megalocitosis, fibrosis hepática y oncosis centrolobulillar regional en el hígado (Copple et al., 2004; Nobre et al., 2004a,b, 2005). Asimismo, se ha reportado la posible presentación de lesiones extrahepáticas en donde pequeñas cantidades de los AP bioactivos pueden entrar a la sangre y ser transportados a otros tejidos, como el parénquima pulmonar o renal (Panter et al., 2019), lo cual podría explicar las alteraciones halladas en pulmón en este estudio (Figura 2B). 

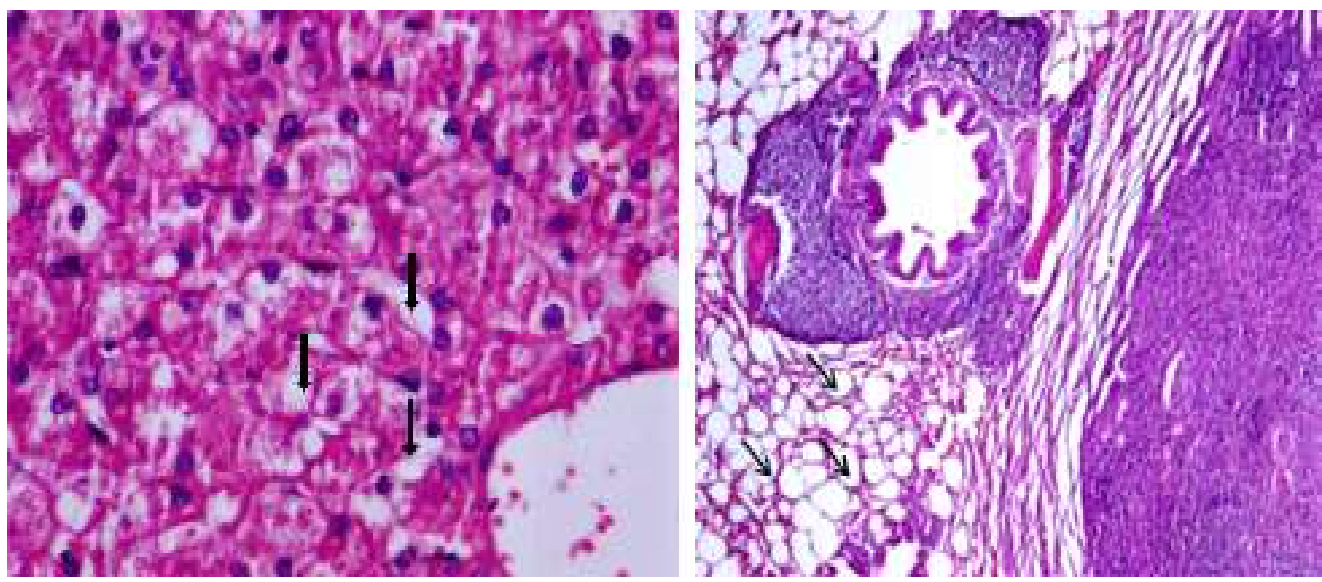

Figura 2. Hallazgos histopatológicos en carneros expuestos al consumo de Crotallaria pallida durante 28 días. A. Alteraciones del parénquima hepático. Las flechas señalan necrosis de hepatocitos con cambio vacuolar severo. B. Alteraciones del parénquima pulmonar. Las flechas señalan engrosamiento de los septos alveolares. 40X, coloración H-E

Los hallazgos histopatológicos pulmonares aquí reportados no coinciden con las lesiones pulmonares descritas por Gardiner et al. (1965), quienes refiere congestión, edema y hemorragias, que según Lee et al. (2005) podría deberse a una hipertensión pulmonar lo que explicaría el edema.

La resistencia de las ovejas a la intoxicación por AP se ha asociado en primera medida con la capacidad de las enzimas hepáticas para desintoxicación, con una baja tasa de producción hepática de pirroles (Cheeke, 1998, 1994); así mismo, Cheeke y Shull (1985) reportaron que existe una mayor producción de EP en bovinos comparados con ovinos. De la misma forma, los microorganismos ruminales pueden desintoxicar los AP consumidos (Culvenor et al., 1984; Duringer et al., 2005). Estas acciones podrían explicar la tolerancia clínica observada en el presente estudio a la ración diaria; pero queda la discusión de que esta misma dosis ha sido reportada por otros autores como tóxica (Nobre et al., 2005; Borelli et al., 2016). Por otro lado, es sabido que las plantas acumulan metabolitos secundarios de acuerdo con consideraciones ontogenéticas de sus procesos adaptativos al agroecosistema y fenómenos medio ambientales preponderantes en el momento de su colecta (Jiménez et al., 2003; Chacón et al., 2013).

\section{Conclusión}

La ingestión diaria por 28 días de dosis bajas de partes aéreas de Crotalaria pallida (hojas y pequeñas ramificaciones) produce intoxicación subcrónica asintomática en ovinos, sin afectar los perfiles hemáticos, hepáticos y renales, pero causando alteraciones histopatológicas en hígado y pulmón.

\section{Literatura Citada}

1. Anjos Leite B, Nobre $V$, Dantas A, Medeiros $R$, Oliveira Neto $T$, Molyneux R, Riet-Correa F. 2010. Poisoning of sheep by seeds of Crotalaria retusa: acquired resistance by continuous administration of low 
doses. Toxicon 55: 28-32. doi: 10.1016/ j.toxicon.2009.06.028

2. Barbosa-Ferreira M, Pfister JA, Gotardo AT, Maiorka P C, Górniak SL. 2011. Intoxication by Senna occidentalis seeds in pregnant goats: prenatal and postnatal evaluation. Exp Toxicol Pathol 63: 263-268. doi: 10.1016/ j.etp.2010.01.004

3. Bernal M. 1986. Flora de Colombia. Bogotá: Imprenta Nacional. 118 p.

4. Boghossian MR, Peixoto PV, Brito MF, Tokarnia CH. 2007. Aspectos clínico-patológicos da intoxicação experimental pelas sementes de Crotalaria mucronata (Fabaceae) em bovinos. Pesq Vet Bras 27: 149-156. doi: 10.1590/ S0100-736X2007000400004

5. Borelli V, Cardoso TC, Biffi CP, Wicpolt N, Ogliari D, Savari T, Sandra $D$, et al. 2016. Intoxicação experimental por folhas de Crotalaria pallida (mucronata) em ovinos. Pesq Vet Bras 36: 935-938. doi: 10.1590/S0100-736X2016001000003

6. Chacón IDLC, Riley-Saldaña CA, González-Esquinca AR. 2013. Secondary metabolites during early development in plants. Phytochem Rev 12: 4764. doi: 10.1007/s11101-012-9250-8

7. Cheeke PR, Shull LR. 1985. Natural toxicants in feeds and poisonous plants. Washington: Westport. $492 \mathrm{p}$.

8. Cheeke PR. 1994. A review of the functional and evolutionary roles of the liver in the detoxification of poisonous plants, with special reference to pirrolizidine alkaloids. Vet Hum Toxicol 36: 240-247.

9. Cheeke PR. 1998. Toxicity and metabolism of pyrrolizidini alkaloids. J Anim Sci 66: 2343-2350. doi: 10.2527/ jas1988.6692343x

10. Chong KY, Tan HT, Corlett RT. 2009. A summary of the total vascular plant flora of Singapore. Gardens' Bull Singapore 63: 197-204.

11. Copple BL, Rondelli CM, Maddox JF, Hoglen NC, Ganey PE, Roth RA. 2004. Modes of cell death in rat liver after monocrotaline exposure. Toxicol Sci 77: 172-182. doi: 10.1093/toxsci/kfh011

12. Culvenor CJ, Jago MV, Peterson JE, Smith LW, Payne AL, Campbell DG, Edgar JA, et al. 1984. Toxicity of Echium plantagineum (Paterson's curse). I. Marginal toxic effects in Merino wethers from long-term feeding. Aust $\mathrm{J}$ Agr Res 35: 293-304.

13. Diaz GJ. 2010. Plantas tóxicas de importancia en salud y producción animal en Colombia. Bogotá: Universidad Nacional de Colombia. $240 \mathrm{p}$.

14. Diaz GJ. 2011. Toxic plants of veterinary and agricultural interest in Colombia. Int J Poisonous Plants Res 1: 1-19.

15. Diaz GJ, Almeida LX, Gardner DR. 2014. Effects of dietary Crotalaria pallida seeds on the health and performance of laying hens and evaluation of residues in eggs. Res Vet Sci 97: 297303. doi: 10.1016/j.rvsc.2014.06.011

16. Diaz GJ. 2015. Toxicosis by plant alkaloids in humans and animals in Colombia. Toxins 7: 12 5408-5416. doi: 10.3390/toxins 7124892

17. Duringer MM, Buhler DR, Craig AM. 2005. Comparison of hepatic in vitro metabolism of the pyrrolizidine alkaloid senecionine in sheep and cattle. Am J Vet Res 65: 1563-1572. doi: 10.2460/ ajvr.2004.65.1563

18. Dusemund B, Nowak N, Sommerfeld $C$, Lindtner $O$, Schäfer B, Lampen A. 2018. Risk assessment of pyrrolizidine alkaloids in food of plant and animal origin. Food Chem Toxicol 115: 63-72. doi: 10.1016/j.fct.2018.03.005

19. Edgar JA, Colegate S, Boppré, M, Molyneux RJ. 2011. Pyrrolizidine alkaloids in food: a spectrum of potential health consequences. Food Addit Contam A 28: 308-324. doi: 10.1080/ 19440049.2010.547520

20. Gardiner MR, Royce R, Bokor A. 1965. Studies on Crotalaria crispata, a newly recognized cause of Kimberly horse disease. J Pathol Bacteriol 89: 4353. doi: 10.1002/path.1700890106. 
21. Grosso DH, Doncel B, Díaz J, Lozano MC. 2011. Análisis costo-beneficio de las medidas instauradas para reducir las pérdidas económicas por plantas tóxicas en explotaciones bovinas de los Llanos orientales colombianos. Rev Colomb Cienc Pecu 24: 553 (Abstract).

22. Hincks JR, Kim HY, Segall HJ, Molyneux RJ, Stermitz FR, Coulombe $\boldsymbol{R} \boldsymbol{A} \boldsymbol{J} \boldsymbol{r}$. 1991. DNA cross-linking in mammalian cells by pyrrolizidine alkaloids: structure-activity relationships. Toxicol Appl Pharm 111: 90-98. doi: 10.1016/0041-008x(91)90137-4

23. Jiménez GS, Ducoing HP, Sosa MR. 2003. La participación de los metabolitos secundarios en la defensa de las plantas. Rev Mex Fitopatol 21: 355-363. doi: 10.15446/abc.v22n3.63405

24. Iason GR, Villalba JJ. 2006. Behavioral strategies of mammal herbivores against plant secondary metabolites: the avoidance-tolerance continuum. J Chem Ecol 32: 6 1115-1132. doi: 10.1007/ s10886-006-9075-2

25. Johnson DD, Libal MC. 1986. Necropsy of sheep and goats. Vet Clin N Am-Food A 2: 129-146. doi: 10.1007/ s10886-006-9075-2

26. Laws L. 1968. Toxicity of Crotalaria mucronata to sheep. Aust Vet J 44: 453455. doi: 10.1111/j.1751-0813.1968.tb08965.x

27. Lee YS, Bryun J, Kim JA, Lee JS, Kim KL, Suh YL, Kim JM, et al. 2005. Monocrotaline-induced pulmonary hypertension correlates with upregulation of connective tissue growth factor expression in the lung. Exp Mol Med 37: 27-35.

28. Lozano MC, Doncel B, Betancourt C, Moreno C. 2010. Ethnobotanical and observational study of toxic plants for bovines in eastern part of Colombia. Toxicol Lett 196: S347. doi: 10.1016/ j.toxlet.2010.03.1097

29. Lozano MC, Diaz GJ. 2013. Poisonous plants for cattle in Colombia: research perspectives. Analecta Vet 33: 33-41. doi: 10.24215/15142590ep.\%2033-41
30. Luna LG 1968. Manual of histologic staining methods of the Armed Forces Institute of Pathology. $3^{\text {rd }}$ ed. New York: McGraw-Hill. 258 p.

31. Madingou OK, Traore A, Souza A, Mounanga MB, Samseny RA, Ouedraogo S, Traore AS. 2016. Preliminary studies of acute and sub-chronic toxicity of the aqueous extract of Guibourtia tessmannii (Harms) J. Leonard stem barks (Caesalpiniaceae) in mice and rats. Asian Pac J Trop Biomed 6: 506-510. doi: 10.1016/ j.apjtb.2016.04.001

32. Mingatto F, Dorta D, dos Santos A, Carvalho I, da Silva C, da Silva V, Uyemura S, et al. 2007. Dehydromonocrotaline inhibits mitochondrial complex. I. A potential mechanism accounting for hepatotoxicity of monocrotaline. Toxicon 50: 724-730. doi: 10.1016/j.toxicon.2007.06.006

33. Mosjidis JA, Wang ML. 2011. Crotalaria. In: Chittaranjan K (ed). Wild crop relatives: genomic and breeding resources. Industrial Crops. SpringerVerlag Berlin Heidelberg 63-69 p

34. [NENT] National Committee for Research Ethics in Science and Technology. 2018. Ethical Guidelines for the Use of Animals in Research: NENT. [Internet]. Disponible en: https:/ /www.forskningsetikk.no/en/guidelines/ science-and-technology/ethicalguidelines-for-the-use-of-animals-inresearch/

35. Nielsen DB, James LF. 1992. The economic impacts of livestock poisonings by plants. In: James LF, Keeler RF, Bailey EM, Cheeke PR, Hegarty MP (eds). Poisonous plants: Proc Third International Symposium. Iowa State, USA.

36. Niwa H, Ogawa T, Yamada K. 1991. Alkylation of nucleoside by dehydromonocrotaline, the putative toxic metabolic of the carcinogenic alkaloid monocrotaline. Tetrahedron Lett 32: 927-930. doi: 10.1016/S0040-4039(00)92122-1 
37. Nobre VMT, Riet-Correa F, Dantas AFM, Tabosa IM, Medeiros RMT, Barbosa Filho JM. 2004a. Intoxication by Crotalaria retusa in ruminants and equidae in the state of Paraíba, northeaster Brazi. In: Plant poisoning and related toxins. Glasgow: CAB International. p 275-279.

38. Nobre VMT, Riet-Correa F, Barbosa Filho JM, Tabosa IM, Vasconcelos JS. 2004b. intoxicação por Crotalaria retusa (Fabaceae) em eqüideos no semiárido da Paraíba. Pesq Vet Bras 24: 132-143. doi: 10.1590/S0100-736X2004000300004

39. Nobre VM, Dantas AF, Riet-Correa F, Barbosa Filho JM, Tabosa IM, Vasconcelos JS. 2005. Acute intoxication by Crotalaria retusa in sheep. Toxicon 45: 347-352. doi: 10.1016/ j.toxicon.2004.11.005

40. Panter K, Welch K, Gardner D. 2019. Poisonous plants: biomar-kers for diagnosis. In: Biomarkers in toxicology. p 627-652.

41. Pessoa CRM, Pessoa AFA, Maia LA, Medeiros RMT, Colegate SM, Barros SS, Soares MP, et al. 2013. Pulmonary and hepatic lesions caused by the dehydropyrrolizidine alkaloid-producing plants Crotalaria juncea and Crotalaria retusa in donkeys. Toxicon 71: 113-120. doi: 10.1016/j.toxicon.2013.05.007

42. Peterson JE, Culvenor CCJ. 1983. Hepatotoxic pyrrolizidine alkaloids. In: Keller RF, Tu AT (eds). Handbook of natural toxins». Marcel Dekker p 638-659.

43. Polhill RM. 1981. Crotalarieae. In: Polhill RM, Raven PH (eds). Advances in legume systematics Part 1. UK: Royal Botanic Gardens. p 399-402.

44. Provenza FD, Pfister JA, Cheney CD. 1992. Mechanisms of learning in diet selection with reference to phytotoxi-cosis in herbivores. J Range Manage 45: 36-45

45. Queiroz G, Lima Ribeiro R, Marques da Costa K, Federico Rodrigues A, Bracarense L, Naylor Lisbôa J. 2013. Intoxicação espontânea por Crotalaria incana em bovinos no norte do estado do Paraná. Semin-Cienc Agrar 34: 823832. doi: 10.5433/1679-0359.2013

46. Radostits OM, Gay CC, Blood DC, Hinchcliff $K W$. 2000. Veterinary medicine. $9^{\text {th }}$ ed. London: WB Saunders. $1877 \mathrm{p}$.

47. Riet-Correa F, Carvalho K, Dantas R, Madeiros R. 2011. Spontaneous acute poisoning by Crotalaria retusa in sheep and biological control of this plant with sheep. Toxicon 58: 606-609. doi: 10.1016/ j.toxicon.2011.09.002

48. Scasta JD, Jorns T, Justin D, Stam B, McClaren M, Calkings C, Stewart W. 2020. Technical note: Toxic plants in sheep diets grazing extensive landscapes: Insights from Fecal DNA metabarcoding. Livest Sci 236: 104002. doi: 10.1016/ j.livsci.2020.104002

49. Souza AC, Hatayde MR, Bechara GH. 1997. Aspectos patológicos da intoxicação de suínos por sementes de Crotalaria spectabilis (Fabaceae). Pesq Vet Bras 17: 12-18. doi: 10.1590/ S0100-736X1997000100003

50. Soares M, Pupin R, Guizelini $C$, Gaspar A, Gomes D, Brumatti R, Lemos R. 2018. Economic losses due to Vernonia rubricaulis poisoning in cattle. Pesq Vet Bras 38: 2217-2223. doi: 10.1590/1678-5150-PVB-6075

51. Tokarnia CH, Döbereiner J. 1982. Intoxicação experimental por Palicourea juruana (Rubiaceae) em bovinos e coelhos. Pesq Vet Bras 2: 17-20. doi: 10.1590/S0100-736X2004000100007

52. Tokarnia CH, Döbereiner J. 1983. Intoxicação experimental por Crotalaria, anagiroides (leg. Papiliono-ideae) em bovinos. Pesq Vet Bras 3:115-123. doi: /10.1590/S0100-736X2004000300004

53. Torres J. 1983. Intoxicación de bovinos por plantas. BAC- Modulo digital [Internet] .Disponible en: https:// repository.agrosavia.co/bitstream/ handle/20.500.12324/23732/ 22929_4072.pdf?sequence $=1 \&$ isAllowed $=\mathrm{y}$ 
54. Wagner JG, Petry TW, Roth RA. 1993. Characterization of monocrotaline pyrrole-induced DNA cross-linking in pulmonary artery endothelium. Am J Physiol 264: L517-L522. doi: 10.1152/ ajplung.1993.264.5.L517

55. Woods J. 2017. On farm euthanasia. Alberta Lamb Producers Agriculture Centre [Internet]. Disponible en: https:// www.ablamb.ca/images/documents/
resources/Sheep-Euthanasia-Manualfinal.pdf

56. Yan CC, Huxtable RJ. 1995. The effect of the pyrrolizidine alkaloids, monocrotaline and trichodesmine, on tissue pyrrole binding and glutathione metabolism in the rat. Toxicon 33: 627634. doi: 10.1016/0041-0101(95)00004-6 